\title{
An Application of the Homotopy Analysis Method to the Transient Behavior of a Biochemical Reaction Model
}

\author{
A. A. M. Arafa ${ }^{1, *}$, S. Z. Rida ${ }^{2}$ and Hegagi Mohamed ${ }^{3}$ \\ ${ }^{1}$ Department of Mathematics and Computer Science, Faculty of Science, Port Said University, Port Said, Egypt \\ ${ }^{2}$ Department of Mathematics, Faculty of Science, South Valley University, Qena, Egypt \\ ${ }^{3}$ Department of Mathematics, Faculty of Science, Aswan University, Aswan, Egypt
}

Published online: 1 Jan. 2014

\begin{abstract}
In this paper, the homotopy analysis method (HAM) is applied to solve fractional transient phase of the Michaelis-Menten reaction. The analytical solution, which is given in the form of a power series, is found to be highly accurate in predicting the behavior of the reaction in the very early stages. The fractional derivatives are described by Caputo's sense. We also present a comparison of the various analytical approximations and a direct numerical solution of this problem. The results of applying this procedure to the studied cases show the high accuracy and efficiency of the approach.
\end{abstract}

Keywords: Homotopy Analysis Method, Fractional Calculus, Michaelis-Menten Biochemical Reaction Model

\section{Introduction}

Mathematical models based on ordinary differential equations appear in the real applications especially in biology because many biological laws and relations appear mathematically in the form of ordinary differential equations. Such models help to understand the underlying principles biological phenomena [1-10]. Here we propose the use of fractional calculus, because of the fact that the realistic modeling of a natural phenomenon does not depend only on the instant time, but also on the history of the previous time which can be successfully achieved by using fractional calculus. In other words, fractional order ordinary differential equations (FODEs) are that they are naturally related to systems with memory which exists in most biological systems. Main claim is that a fractional model can give a more realistic interpretation of natural phenomena. However, numerical methods commonly need large computation work and have round-off error problems. The Homotopy Analysis Method (HAM) which first proposed in 1992 by liao [11] is successful method to find the exact analytical solutions for linear and nonlinear problem. This method has been successfully applied into physics, chemical, biology, engineering fields and science [12-16]. In this paper, we present a solution of a more general model of an enzyme-catalyzed reaction model:

$$
\begin{array}{r}
D_{t}^{\alpha_{1}} a=-a+(\beta-\alpha) y+a y, \\
D_{t}^{\alpha_{2}} y=\frac{1}{\varepsilon}(a-\beta y-a y)
\end{array}
$$

Subject to the initial conditions

$$
a(0)=1, y(0)=0
$$

Where $a$ is a concentrations of substrate, $y$ is an intermediate complex between enzyme and $\alpha, \beta, \varepsilon$ are dimensionless parameters. For more details of the standard model see [17-18].

\section{Basic definitions}

In this section, we mention the basic definitions of the fractional calculus.

Definition 1 The Riemann-Liouvlle fractional integral operator $\left(J^{\alpha}\right)$ of order $\alpha \geq 0$, of a function $h \in c_{\mu}, \mu \geq-1$ Is defined as;

$$
\begin{gathered}
J^{\alpha} h(t)=\frac{1}{\Gamma(\alpha)} \int_{0}^{t}(t-\tau)^{\alpha-1} h(\tau) d \tau \quad(\alpha \succ 0) \\
J^{0} h(t)=h(t)
\end{gathered}
$$

\footnotetext{
*Corresponding author e-mail: anaszi2@yahoo.com
} 
$\Gamma(\alpha)$ is the well-known gamma function some of the properties of the operator $\left(J^{\alpha}\right)$, Which we will need here, are as follows. for $h \in c_{\mu}, \mu \geq-1, \alpha, \beta \geq 0$ and $\gamma \geq-1$ :

$$
\begin{gathered}
\text { (1) } J^{\alpha} J^{\beta} h(t)=J^{\alpha+\beta} h(t), \\
\text { (2) } J^{\alpha} J^{\beta} h(t)=J^{\beta} J^{\alpha} h(t), \\
\text { (3) } J^{\alpha} t^{\gamma}=\frac{\Gamma(\gamma+1)}{\Gamma(\gamma+\alpha+1)} t^{\alpha+\gamma} \\
J^{\alpha} e^{a t}=t^{\alpha} \sum_{k=0}^{\infty} \frac{(a t)^{k}}{\Gamma(\alpha+k+1)}
\end{gathered}
$$

Definition 2 The fractional derivative of $f(x)$ in the Caputo sense is defined as:

$$
D^{\alpha} f(x)=J^{n-\alpha} D^{n} f(x)=\frac{1}{\Gamma(n-\alpha)} \int_{0}^{x}(x-t)^{n-\alpha-1} f^{(n)}(t) d t,
$$

For $n-1<\alpha<n, n \in N, \mathrm{x}>0$.for the Caputo derivative we have $D^{\alpha} C=0, \mathrm{C}$ is constant and

$$
D^{\alpha} t^{m}=\left\{\begin{array}{lr}
0 & m \leq \alpha-1 \\
\frac{\Gamma(m+1)}{\Gamma(m-\alpha+1)}, & m>\alpha-1
\end{array}\right.
$$

Definition 3 For $\mathrm{n}$ to be the smallest integer that exceeds $\alpha$, the Caputo fractional derivatives of order $\alpha>0$ is defined as

$D^{\alpha} u(x, t)=\frac{\partial^{\alpha} u(x, t)}{\partial t^{\alpha}}= \begin{cases}\frac{1}{\Gamma(n-\alpha)} \int_{0}^{t}(t-\tau)^{n-\alpha+1} \frac{\partial^{\alpha} u(x, \tau)}{\partial \tau^{\alpha}} d \tau, & \text { for } n-1<\alpha \leq n, \\ \frac{\partial^{n} u(x, t)}{\partial t^{\alpha}}, & \text { for } \alpha=n \in N .\end{cases}$

\section{Homotopy analysis method}

Let us consider the following system of differential equation

$$
N_{i}\left[u_{1}(r, t), \ldots, u_{n}(r, t)\right]=0, i=1,2,3, \ldots, n .
$$

Subject to the following initial conditions:

$$
u_{k}(r, t)=c_{k}, k=1,2,3, \ldots, n .
$$

where $N_{i}$ nonlinear operators that represent the whole equations, $r$ and $\mathrm{t}$ are denote the independent variables and $u_{i}(r, t)$ are unknowns function respectively. By means of generalizing the traditional homotopy method, Liao [11] constructed the so-called zero-order deformation equations for $\mathrm{i}=1,2, \ldots, \mathrm{n}$,

$$
(1-q) \varphi_{i}\left[\phi_{i}(r, t ; q)-u_{i 0}(r, t)\right]=q h_{i} H_{i}(r, t) N_{i}\left[\phi_{1}(r, t ; q), \ldots, \phi_{n}(r, t ; q)\right],
$$

where $\mathrm{q} \in[0,1]$ is the embedding parameter, $h_{i} \neq 0$ are non-zero auxiliary parameters for $H_{i}(r, t) \neq 0$ are non-zero auxiliary functions, $\varphi_{i}=D_{t}^{\alpha_{i}}(n-1<\alpha \leq n)$ are auxiliary linear operator with the following property for $\mathrm{i}=1,2,3, \ldots, \mathrm{n}$

$$
\varphi_{i}\left[\phi_{i}(r, t)\right]=0 w h e n \phi_{i}(r, t)=0
$$

$u_{i 0}(r, t)$ are initial guess of $u_{i}(r, t), u_{i}(r, t ; q)$ are unknown function, respectively. It is important, that one has great freedom to choose auxiliary things in HAM. Obviously, when $\mathrm{q}=0$ and $\mathrm{q}=1$, it holds

$$
\phi_{i}(r, t ; 0)=u_{i 0}(r, t), \phi_{i}(r, t ; 1)=u_{i}(r, t), i=1,2, \ldots, n,
$$

respectively. Thus, as q increases from 0 to 1 , the solution $\phi_{i}(r, t, q)$ varies from the initial guesses $u_{i 0}(r, t$,$) to the$ solution $u_{i}(r, t$,$) . Expanding \phi_{i}(r, t, q)$ in Taylor series with respect to $\mathrm{q}$, we have

$$
\phi_{i}(r, t, q)=u_{i 0}(r, t)+\sum_{m=1}^{+\infty} u_{i m}(r, t) q^{m}, i=1,2, \ldots, n .
$$

where

$$
u_{i m}(r, t)=\left.\frac{1}{m !} \frac{\partial^{m} \phi_{i}(r, t ; q)}{\partial q^{m}}\right|_{q=0}, i=1,2, \ldots, n .
$$

If the auxiliary linear operator, the initial guess, the auxiliary parameter $\mathrm{h}$, and the auxiliary function are so properly chosen, the series Eq. (16) converges at $\mathrm{q}=1$, then we have

$$
\phi_{i}(r, t, q)=u_{i 0}(r, t)+\sum_{m=1}^{+\infty} u_{i m}(r, t), i=1,2, \ldots, n .
$$

Define

the vector $\vec{u}_{n}^{i}=\left\{u_{i 0}(r, t), u_{i 1}(r, t), u_{i 2}(r, t), \ldots, u_{i n}(r, t)\right\}, \mathrm{i}=1,2, \ldots, \mathrm{n}$, Differentiating Eq. (13) times with respect to the embedding parameter $\mathrm{q}$ and then setting $\mathrm{q}=0$ and finally dividing them by $\mathrm{m}$ !, we obtain the mth-order deformation equation for $\mathrm{i}=1,2, \ldots, \mathrm{n}$,

$\varphi_{i}\left[u_{i m}(r, t)-u_{i m-1}(r, t)\right]=h_{i} H_{i}(r, t) R_{i m}(\underset{m-1}{\vec{u} 1}(r, t), \ldots, \underset{m-1}{\vec{u}}(r, t))$

where

$$
\begin{aligned}
& R_{i m}\left(\underset{m-1}{\vec{u}_{1}^{1}}(r, t), \ldots, \underset{m-1}{\vec{u}_{n}^{n}}(r, t)\right), \\
& =\left.\frac{1}{(m-1) !} \frac{\partial^{m-1} N_{i}\left[\phi_{1}(r, t ; q), \ldots, \phi_{n}(r, t ; q)\right]}{\partial q^{m-1}}\right|_{q=0}
\end{aligned}
$$

And

$$
\chi_{m}= \begin{cases}0 & m \leq 1, \\ 1 & m>1 .\end{cases}
$$

Applying the Riemann-Liouville integral operator $J^{\alpha_{i}}$ on both side of Eq. (19), we have

$$
u_{i m}=\chi_{m} u_{i m-1}+h_{i} H_{i}(r, t) J^{\alpha_{i}}\left[R_{i m}\left(\underset{m-1}{\overrightarrow{u_{i}}}(r, t), \ldots, \underset{m-1}{\vec{u}}(r, t)\right)\right] .
$$

In this way, it is easily to obtain $u_{i, m}(r, t)$ form $\geq 1$, at Mth order, we have

$$
u_{i}(r, t)=\sum_{m=0}^{\infty} u_{i m}(r, t), i=1,2,3, \ldots, n .
$$

We get an accurate approximation of the original Eq.(11). 


\section{Applications of the HAM}

In this section, we apply the homotopy analysis method to solve the system

$$
\begin{gathered}
D_{t}^{\alpha_{1}} a=-a+(\beta-\alpha) y+a y, \\
D_{t}^{\alpha_{2}} y=\frac{1}{\varepsilon}(a-\beta y-a y),
\end{gathered}
$$

Subject to the initial conditions

$$
a(0)=1, y(0)=0 .
$$

We choose the linear operator

$$
\begin{array}{llrl}
L_{1}[\phi(x, y, t ; q)] & =D_{t}^{\alpha_{1}} \phi(x, y, t ; q), & & L_{1}^{-1}=J^{\alpha_{1}} \phi(x, y, t ; q) \\
L_{2}[\phi(x, y, t ; q)] & =D_{t}^{\alpha_{2}} \phi(x, y, t ; q), & & L_{2}^{-1}=J^{\alpha_{2}} \phi(x, y, t ; q)
\end{array}
$$

With the property $L_{1}[c]=L_{2}[c]=0$, where $\mathrm{c}$ is constant. We now define a nonlinear operator as:

$N_{1}\left[\phi_{1}(x, y, t ; q)\right]=D_{t}^{\alpha_{1}} \phi_{1}(x, y, t)+\phi_{1}(x, y, t)-(\beta-\alpha) \phi_{2}(x, y, t)-\phi_{1}(x, y, t) \phi_{2}(x, y, t)$, $N_{2}\left[\phi_{2}(x, y, t ; q)\right]=D_{t}^{\alpha_{2}} \phi_{2}(x, y, t)-\frac{1}{\varepsilon} \phi_{1}(x, y, t)+\frac{\beta}{\varepsilon} \phi_{2}(x, y, t)+\frac{1}{\varepsilon} \phi_{1}(x, y, t) \phi_{2}(x, y, t)$

Using above definition, with assumption $H(\tau)=1$ we construct the zero order

deformation equation

$$
\begin{aligned}
(1-q) L_{1}\left[\phi_{1}(x, y, t ; q)-a_{0}(x, y, t)\right] & =q h N_{1}\left[\phi_{1}(x, y, t ; q)\right], \\
(1-q) L_{2}\left[\phi_{2}(x, y, t ; q)-y_{0}(x, y, t)\right] & =q h N_{2}\left[\phi_{2}(x, y, t ; q)\right]
\end{aligned}
$$

Obviously, when $\mathrm{q}=0$ and $\mathrm{q}=1$,

$$
\begin{array}{ll}
\phi_{1}(x, y, t ; 0)=a_{0}(x, y, t) & \quad \phi_{1}(x, y, t ; 1)=a(x, y, t) \\
\phi_{2}(x, y, t ; 0)=y_{0}(x, y, t) \quad, & \phi_{2}(x, y, t ; 1)=y(x, y, t)
\end{array}
$$

Thus, we obtain the mth-order deformation equations

$$
\begin{aligned}
& L_{1}\left[a_{m}-\chi_{m} a_{m-1}\right]=h R_{m}(\underset{m-1}{\vec{a}}), \\
& L_{2}\left[y_{m}-\chi_{m} y_{m-1}\right]=h R_{m}(\underset{m-1}{\vec{y}})
\end{aligned}
$$

where

$$
\begin{aligned}
& R_{m}(\underset{m-1}{\vec{a}})=D_{t}^{\alpha_{1}} a_{m-1}+a_{m-1}-(\beta-\alpha) y_{m-1}-\sum_{i=0}^{m-1} a_{i} y_{m-1-i}, \\
& R_{m}(\underset{m-1}{\vec{y}})=D_{t}^{\alpha_{2}} y_{m-1}-\frac{1}{\varepsilon} a_{m-1}+\frac{\beta}{\varepsilon} y_{m-1}+\frac{1}{\varepsilon} \sum_{i=0}^{m-1} a_{i} y_{m-1-i}
\end{aligned}
$$

Now, the solution of the mth-order deformation equation (23)

$$
\begin{aligned}
& a_{m}=\chi_{m} a_{m-1}+h J^{\alpha_{1}}\left[R_{m} \underset{m-1}{\vec{a}}\right], \\
& y_{m}=\chi_{m} y_{m-1}+h J^{\alpha_{2}}\left[R_{m}(\underset{m-1}{\vec{y}}]\right.
\end{aligned}
$$

From equation (31) we have,

$$
\begin{aligned}
& a_{m}=\chi_{m} a_{m-1}+h J^{\alpha_{1}}\left[D_{t}^{\alpha_{1}} a_{m-1}+a_{m-1}-(\beta-\alpha) y_{m-1}\right. \\
& \left.-\sum_{i=0}^{m-1} a_{i} y_{m-1-i}\right] \\
& y_{m}=\chi_{m} y_{m-1}+h J^{\alpha_{2}}\left[D_{t}^{\alpha_{2}} y_{m-1}-\frac{1}{\varepsilon} a_{m-1}+\frac{\beta}{\varepsilon} y_{m-1}\right. \\
& \left.+\frac{1}{\varepsilon} \sum_{i=0}^{m-1} a_{i} y_{m-1-i}\right]
\end{aligned}
$$

Consequently, the first few terms of the HAM

$$
\begin{gathered}
a_{0}=1, y_{0}=0, \\
a_{1}=h \frac{t^{\alpha_{1}}}{\Gamma\left(\alpha_{1}+1\right)}, y_{1}=-\frac{h}{\varepsilon} \frac{t^{\alpha_{2}}}{\Gamma\left(\alpha_{2}+1\right)}, \\
a_{2}=\left(h^{2}+h\right) \frac{t^{\alpha_{1}}}{\Gamma\left(\alpha_{1}+1\right)}+h^{2} \frac{t^{2 \alpha_{1}}}{\Gamma\left(2 \alpha_{1}+1\right)}+\frac{h^{2}}{\varepsilon}(\beta-\alpha+1) \frac{t^{\alpha_{1}+\alpha_{2}}}{\Gamma\left(\alpha_{1}+\alpha_{2}+1\right)}, \\
y_{2}=-\frac{1}{\varepsilon}\left(h^{2}+h\right) \frac{t^{\alpha_{2}}}{\Gamma\left(\alpha_{2}+1\right)}-\frac{h^{2}}{\varepsilon^{2}}(\beta+1) \frac{t^{2 \alpha_{2}}}{\Gamma\left(2 \alpha_{2}+1\right)}-\frac{h^{2}}{\varepsilon} \frac{t^{\alpha_{1}+\alpha_{2}}}{\Gamma\left(\alpha_{1}+\alpha_{2}+1\right)}, \\
a_{3}=\left(h^{3}+2 h^{2}+h\right) \frac{t^{\alpha} \alpha_{1}}{\Gamma\left(\alpha_{1}+1\right)}+\left(2 h^{3}+2 h^{2}\right) \frac{t^{2 \alpha_{1}}}{\Gamma\left(2 \alpha_{1}+1\right)}+h^{3} \frac{t^{3 \alpha_{1}}}{\Gamma\left(3 \alpha_{1}+1\right)}+ \\
\quad \frac{(\beta-\alpha+1)}{\varepsilon}\left(2 h^{3}+2 h^{2}\right) \frac{t^{\alpha_{1}+\alpha_{2}}}{\Gamma\left(\alpha_{1}+\alpha_{2}+1\right)}+\frac{h^{3}}{\varepsilon}(\beta-\alpha+1)(\beta+1) \frac{t^{\alpha_{1}+2 \alpha_{2}}}{\Gamma\left(\alpha_{1}+2 \alpha_{2}+1\right)} \\
+\frac{h^{3}}{\varepsilon}\left\{2(\beta-\alpha+1)+\frac{\Gamma\left(\alpha_{1}+\alpha_{2}+1\right)}{\Gamma\left(\alpha_{1}+1\right) \Gamma\left(\alpha_{2}+1\right)}\right\} \frac{t^{2 \alpha_{1}+\alpha_{2}}}{\Gamma\left(2 \alpha_{1}+\alpha_{2}+1\right)} \\
y_{3}=-\frac{1}{\varepsilon}\left(h^{3}+2 h^{2}+h\right) \frac{t^{\alpha_{2}}}{\Gamma\left(\alpha_{2}+1\right)}-\frac{(\beta+1)}{\varepsilon^{2}}\left(2 h^{3}+2 h^{2}\right) \frac{t^{2} \alpha_{2}}{\Gamma\left(2 \alpha_{2}+1\right)}- \\
\frac{h^{3}}{\varepsilon^{3}}(\beta+1)^{2} \frac{t^{3} \alpha_{2}}{\Gamma\left(3 \alpha_{1}+1\right)}-\frac{1}{\varepsilon}\left(2 h^{3}+2 h^{2}\right) \frac{t^{\alpha_{1}+\alpha_{2}}}{\Gamma\left(\alpha_{1}+\alpha_{2}+1\right)}-\frac{h^{3}}{\varepsilon} \frac{t^{2} \alpha_{1}+\alpha_{2}}{\Gamma\left(\alpha_{1}+2 \alpha_{2}+1\right)} \\
-\frac{h^{3}}{\varepsilon^{2}}\left\{2(\beta-\alpha+1)+\frac{\Gamma\left(\alpha_{1}+\alpha_{2}+1\right)}{\Gamma\left(\alpha_{1}+1\right) \Gamma\left(\alpha_{2}+1\right)}\right\} \frac{t^{\alpha_{1}+2 \alpha_{2}}}{\Gamma\left(\alpha_{1}+2 \alpha_{2}+1\right)}
\end{gathered}
$$

Finally, we have

$$
a(x, y, t)=\sum_{m=0}^{\infty} a_{m}(x, y, t), y(x, y, t)=\sum_{m=0}^{\infty} y_{m}(x, y, t)
$$

Then

$$
\begin{aligned}
& a_{m}=1+\left(h^{3}+3 h^{2}+3 h\right) \frac{t^{\alpha_{1}}}{\Gamma\left(\alpha_{1}+1\right)}+\left(2 h^{3}+3 h^{2}\right) \frac{t^{2 \alpha_{1}}}{\Gamma\left(2 \alpha_{1}+1\right)}+h^{3} \frac{t^{3 \alpha_{1}}}{\Gamma\left(3 \alpha_{1}+1\right)}+ \\
& \quad \frac{(\beta-\alpha+1)}{\varepsilon}\left(2 h^{3}+3 h^{2}\right) \frac{t^{\alpha} \alpha_{1}+\alpha_{2}}{\Gamma\left(\alpha_{1}+\alpha_{2}+1\right)}+\frac{h^{3}}{\varepsilon^{2}}(\beta-\alpha+1)(\beta+1) \frac{t^{\alpha_{1}+2 \alpha_{2}}}{\Gamma\left(\alpha_{1}+2 \alpha_{2}+1\right)} \\
& \quad+\frac{h^{3}}{\varepsilon}\left\{2 \beta-\alpha+2+\frac{\Gamma\left(\alpha_{1}+\alpha_{2}+1\right)}{\Gamma\left(\alpha_{1}+1\right) \Gamma\left(\alpha_{2}+1\right)}\right\} \frac{t^{2} \alpha_{1}+\alpha_{2}}{\Gamma\left(2 \alpha_{1}+\alpha_{2}+1\right)}+\ldots
\end{aligned}
$$

$$
\begin{aligned}
& y_{m}=-\frac{1}{\varepsilon}\left(h^{3}+3 h^{2}+3 h\right) \frac{t^{\alpha_{2}}}{\Gamma\left(\alpha_{2}+1\right)}-\frac{(\beta+1)}{\varepsilon^{2}}\left(2 h^{3}+3 h^{2}\right) \frac{t^{2} \alpha_{2}}{\Gamma\left(2 \alpha_{2}+1\right)}- \\
& \quad \frac{h^{3}}{\varepsilon^{3}}(\beta+1)^{2} \frac{t^{3} \alpha_{2}}{\Gamma\left(3 \alpha_{1}+1\right)}-\frac{1}{\varepsilon}\left(2 h^{3}+3 h^{2}\right) \frac{t^{\alpha_{1}+\alpha_{2}}}{\Gamma\left(\alpha_{1}+\alpha_{2}+1\right)}-\frac{h^{3}}{\varepsilon} \frac{t^{2} \alpha_{1}+\alpha_{2}}{\Gamma\left(2 \alpha_{1}+\alpha_{2}+1\right)} \\
& \quad-\frac{h^{3}}{\varepsilon^{2}}\left\{2 \beta-\alpha+2+\frac{\Gamma\left(\alpha_{1}+\alpha_{2}+1\right)}{\Gamma\left(\alpha_{1}+1\right) \Gamma\left(\alpha_{2}+1\right)}\right\} \frac{t^{\alpha_{1}+2 \alpha_{2}}}{\Gamma\left(\alpha_{1}+2 \alpha_{2}+1\right)}-\ldots
\end{aligned}
$$

Equations (28) and (29) are solutions of fractional enzyme-catalyzed reaction model. For the purpose of comparing the analytical and numerical results, the following parameter values are used: $\alpha=0.375, \beta=1$ and $\varepsilon=0.1$ which given from [?]. Respectively, Figure 1 shows that the convergence region of the series solution $-0.5 \leq h \leq-1.2$ and Figure 2 shows that in the initial stages, the concentration of the complex rises until it reaches a maximum. The results show that, our results when $\alpha_{1}=\alpha_{2}=1$ are agreement with the result in [19]. See Table1 and Figure 1

\section{Conclusions}

We employ the HAM for finding the solutions of intermediate complex biochemical reaction model, and compare this solution with analytical and numerical solutions for different times. From the results we seen that HAM is a very powerful and efficient technique in finding analytical solutions for wide classes of systems of equations. They also do not require large computer memory, the results show that HAM is powerful mathematical tool for solving linear and nonlinear equations 
Table 1: Comparison of the Homotopy Analysis Method, Direct Numerical Solutions and the Power Series for the Concentration of the Intermediate Complex, with $\alpha=0.375, \beta=1, \varepsilon=0.1$ and $h=-0.76$.

\begin{tabular}{|l|l|l|l|}
\hline $\mathrm{t}$ & $\begin{array}{l}\text { Homotopy } \\
\text { analysis } \\
\text { solution } \\
y_{H A M}\end{array}$ & $\begin{array}{l}\text { Direct } \\
\text { numerical } \\
\text { solution } \\
y_{N}\end{array}$ & $\begin{array}{l}\text { Power series } \\
\text { solution } \\
y_{A}\end{array}$ \\
\hline 0.05 & 0.310507 & 0.310751 & 0.310727 \\
\hline 0.075 & 0.37587 & 0.380089 & 0.379242 \\
\hline 0.10 & 0.423122 & 0.421519 & 0.411202 \\
\hline
\end{tabular}

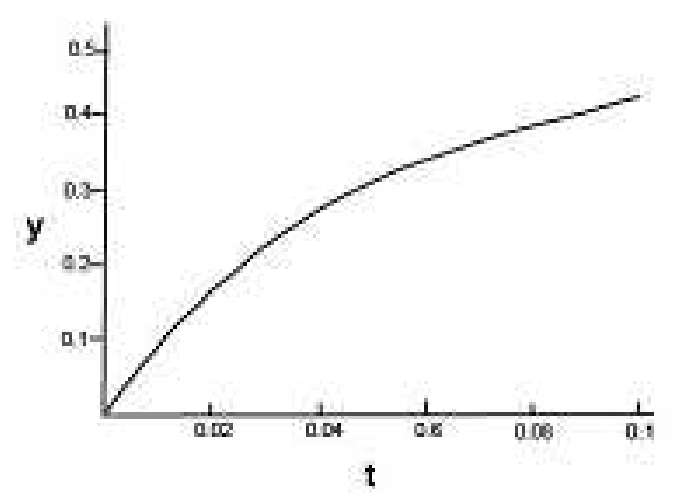

Fig. 1: The h curve of $y^{\prime}(0)$ obtained from HAM approximation solution of Eq.(29)

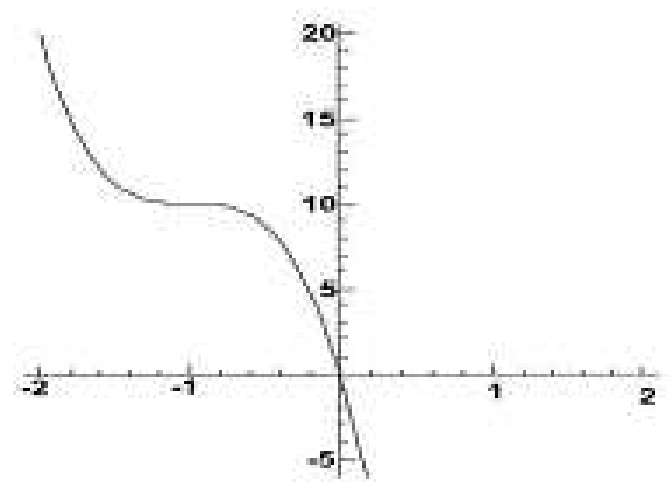

Fig. 2: The HAM solution for intermediate complex for Eq.(29) of $y(t)$ in case of $h=-0.76, \alpha_{1}=\alpha_{2}=1$, and parameter values are used: $\alpha=0.375, \beta=1, \varepsilon=0.1$

\section{References}

[1] A. A. M. Arafa, Fractional Differential Equations in Description of Bacterial Growth, Differential Equations \& Dynamical Syststem, 21, 205-214 (2013).

[2] A.A. M. Arafa, S.Z. Rida, M. Khalil, Approximate analytical solutions of Schnakenberg systems by homotopy analysis method, Applied Mathematical Modeling. 37, 2189-2196 (2013).
[3] A.A.M. Arafa,S.Z. Rida, A.A. Mohammadein,and H.M. Ali, Solving Nonlinear Fractional Differential Equation by Generalized Mittag-Leffler Function Method, Commun. Theor. Phys. 59, 661-663 (2013).

[4] A.A. M. Arafa, S.Z. Rida, Numerical modeling for some generalized coupled nonlinear evolution equations, Mathematical and Computer Modelling. 56, 268-277 (2012).

[5] A.A. M. Arafa, S.Z. Rida, H. Mohamed, Approximate analytical solutions of Schnakenberg systems by homotopy analysis method, Applied Mathematical Modelling. 36, 47894796 (2012).

[6] A.A. M. Arafa, S.Z. Rida, and M. Khalil, Fractional modeling dynamics of HIV and $C D 4^{+}$T-cells during primary infection, Nonlinear Biomedical Physics. 61 (2012) doi:10.1186/17534631-6-1.

[7] A. A. M. Arafa, Series Solutions of Time-Fractional HostParasitoid Systems, J Stat Phys 145, 1357-1367 (2011).

[8] S. Z. Rida and A. A. M. Arafa, New Method for Solving Linear Fractional Differential Equations, International journal of differential equations., Article ID 814132 (2011) doi:10.1155/2011/814132.

[9] K. B. Oldham, J. Spanier, The fractional calculus, New York: Academic Press, 99, (1974).

[10] I. Podlubny, Fractional differential equations, New York: Academic Press; (1999).

[11] S. J. Liao, The proposed homotopy analysis technique for the solution of nonlinear problems, PhD thesis, Shanghai Jiao Tong University; (1992).

[12] S. J. Liao, A. Campo, Analyticsolutions of the temperature distribution in Blasius viscous flow problems, $J$ Fluid Mech. 453, 411-25 (2002).

[13] M. Ayub, A. Rasheed, T. Hayat, Exactflow of a third grade fluid past a porous plate using homotopy analysis method, Int J Eng Sci., 41, 2091-103 (2003).

[14] S. Abbasbandy, Homotopy analysis method for heat radiation equations, Int Comm Heat Mass Transfer. 34, 380 (2007).

[15] S. Abbasbandy, The application of homotopy analysis method to solve a generalized Hirota-Satsuma coupled KdV equation, Phys Lett A. 361, 478-483 (2007).

[16] S. Abbasbandy, Approximate solution for the nonlinear model of diffusion and reaction in porous catalysts by means of the homotopy analysis method, Chem Eng $J$. doi:10.1016/j.cej, (2007).

[17] Hashim, M.S.H. Chowdhury, S. Mawa, On multistage homotopy-perturbation method applied to nonlinear biochemical reaction model, Chaos, Solitons and Fractals. 36, 823-827 (2008).

[18] L. A. Segel, Mathematical Models in Molecular and Cellular Biology, Cambridge Univ. Press, Cambridge, (1980).

[19] A. K. Sen, An application of the adomian decomposition method to the transient behavior of a model biochemical reaction, Journal of mathematical analysis and applications. 131, 232-245 (1988).

[20] M. Dixon, E. C. Webb, The Enzymes, Academic Press, New York, (1964).

[21] H. Gutfreund, Steps in the formation and decomposition of some enzyme-substrate complexes, Discuss. Faraday Sot. 20, 167-173 (1955). 


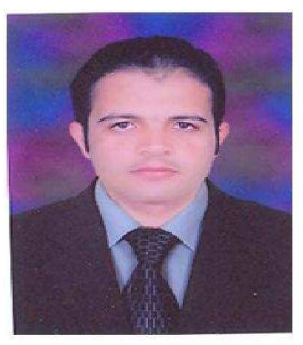

A. A. M. Arafa is a lecturer of Mathematics at Port Said University, Faculty of Science. He is referee and Editor of several international journals in the frame of pure and applied mathematics. His main research interests are: Fractional Calculus and applications.

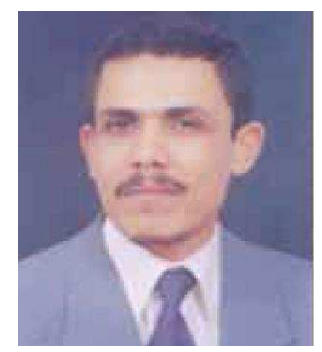

S. Z. Rida is Professor and Head of Mathematics Department at Faculty of Science, South Valley University. He is referee and Editor of several international journals in the frame of pure and applied mathematics. His main research interests are: Fractional Calculus and applications

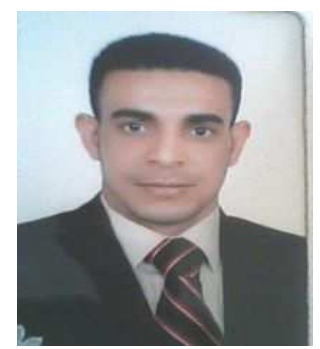

Hegagi Mohamed
is an Assistant Lecturer
of Mathematics at Aswan
University, Faculty of
Science. He published
some papers in international
journals in the frame of pure
and applied mathematics.
His main research interests
are: Fractional Calculus
and applications

